\title{
Gauge invariant information concerning quantum channels
}

\author{
Łukasz Rudnicki ${ }^{1,2}$, Zbigniew Puchała ${ }^{3,4}$, and Karol Życzkowski ${ }^{2,4}$ \\ ${ }^{1}$ Institute for Theoretical Physics, University of Cologne, Zülpicher Straße 77, D-50937, Cologne, Germany \\ ${ }^{2}$ Center for Theoretical Physics, Polish Academy of Sciences, Al. Lotników 32/46, 02-668 Warsaw, Poland \\ ${ }^{3}$ Institute of Theoretical and Applied Informatics, Polish Academy of Sciences, ulica Bałtycka 5, 44-100 Gliwice, Poland \\ ${ }^{4}$ Faculty of Physics, Astronomy and Applied Computer Science, Jagiellonian University, ul. Łojasiewicza 11, 30-348 Kraków, \\ Poland
}

March 16, 2018

Motivated by the gate set tomography we study quantum channels from the perspective of information which is invariant with respect to the gauge realized through similarity of matrices representing channel superoperators. We thus use the complex spectrum of the superoperator to provide necessary conditions relevant for complete positivity of qubit channels and to express various metrics such as average gate fidelity.

\section{Introduction}

Gauge symmetries are among the most influential concepts of modern physics. They provide suitable underlying structures for all known field theories such as Electrodynamics, General Relativity or Yang-Mills theory. Most importantly, while working with a theory equipped with a gauge, one becomes guided to meaningful quantities, namely those which are gauge invariant (do not depend on the gauge degrees of freedom).

Let us change a perspective for a moment and focus on the following problem. An unknown square matrix $\Phi$ is provided to us through a similar matrix $M$

$$
\Phi=X M X^{-1} \quad \text { or } \quad M=X^{-1} \Phi X .
$$

Let $X$ be yet another, more or less arbitrary, invertible matrix of the same size as $\Phi$ and $M$. If $X$ is further assumed to belong to a certain group, one can be tempted to call (1) the gauge transformation. It is important to stress that this conceptual association is right only if we cannot get Łukasz Rudnicki: rudnicki@cft.edu.pl the full information concerning $X$, so that both $\Phi$ and $M$ are truly equivalent.

Such a situation is inherent to gate set tomography (GST) [1, 2], a modern view [3] on quantum process tomography [4], in which gauge-related terminology is common in use. In this scheme, the matrix $\Phi$ is a superoperator which represents a true quantum channel, while $M$ is its reconstruction based on experimental data. The matrix $X$ is a symmetry, which leaves invariant all measured probabilities.

In fact, in GST one simultaneously deals with a, so called, gate set $\mathcal{G}$ consisting of several gates (quantum channels) $\Phi_{k}$, an initial state $\rho$ and a final generalized measurement with two outcomes, $\{E, \mathbb{1}-E\}$ - all being unknown. During the estimation procedure one must reconstruct all the gates in question, as well as the initial state and the applied measurement. The crucial conceptual difference, with respect to standard quantum (process) tomography, is that linearly independent sets of initial states and final measurements are obtained through sequences of gates from $\mathcal{G}$ applied to the single initial state and the single final measurement. Note that the above idea was also considered directly in full process[5] and state-tomography [6]; in the latter case in a somewhat implicit way. The discussed novelty, while being a source of several advantages of GST over older approaches, is also responsible for the "gauge" ambiguity, since all the probabilities measured during the associated experiments are of the form:

$$
\left\langle\left\langle E\left|\Phi_{k_{1}} \Phi_{k_{2}} \cdots \Phi_{k_{n}}\right| \rho\right\rangle\right\rangle,
$$

where $|\cdot\rangle\rangle$ and $\langle\langle\cdot|$ denote (co)vector representations of involved matrices, to be multiplied by 
the superoperators. Clearly [1,3], the probabilities (2) remain the same if instead of $\left.\Phi_{k},|\rho\rangle\right\rangle$ and $\left\langle\langle E|\right.$ one provides $\left.\left.M_{k}=X^{-1} \Phi_{k} X, X^{-1} \mid \rho\right\rangle\right\rangle$ and $\langle\langle E| X$.

It seems, however, that so far the "gauge degree of freedom" of the GST, even though words like "transformation" or "invariance" are among its semantic collocates [7], has not been treated as a serious player. While working with Electrodynamics, for instance, one can either consider a vector potential in a fixed gauge (less preferable option) or resort to gauge invariant quantities such as the electromagnetic field. In GST, the first path is usually followed, so that $X$ becomes fixed by means of an optimization procedure. The figure of merit to be optimized might in principle be a matter of choice or convenience, however, it usually is expected to minimize the discrepancy between $\mathcal{G}$ and a predefined target gate set $\mathcal{G}^{\prime}$ [7]. On the one hand, taking this approach is not surprising since the GST gauge, in its formal aspects, is only a shadow of its field-theoretic counterpart. There is no room for structures such as an underlying manifold (spacetime), gauge connection, curvature, Killing vectors, etc. On the other hand, by fixing the gauge in a way which makes $\mathcal{G}$ possibly close to $\mathcal{G}^{\prime}$, one likely underestimates the so-called QCVV (quantum characterization, verification, and validation) metrics, such as average gate fidelity [8] or the diamond norm [9], relevant for fault-tolerant quantum computing.

A broader context of QCVV enlightens yet another issue. A genuine gauge is not only a degree of freedom one cannot control (this is fulfilled in GST) but also one which, being inherent to a physical phenomenon, cannot be avoided. The latter property is not clearly satisfied by the GST gauge. Even though one argues that the level of quantum control, noise reduction etc. necessary to make quantum process tomography faithful (can one prepare an orthogonal set of input states in a fully controlled way?) is by far beyond the current technology [1], there is no fundamental obstacle in achieving the desired level in the future. Moreover, there are other, complementary methods such as randomized benchmarking $[10,11,12,13]$, which allow for quality assessment of the implemented gates. Thus, even though there is no room for a proper, rich GST gauge theory, the main aspect brought by the gauge - the necessity to rely only on gauge invariant quantities - seems rather indispensable.

The aim of this paper is twofold. First of all, we make first attempts to discuss about properties of quantum channels by means of gauge-invariant information, showing natural limitations appearing in that strategy. In more detail, we provide in Section 3 gauge invariant, necessary criteria for complete positivity of qubit $(d=2)$ quantum channels and discuss in Section 4 gauge invariant expressions and bounds (in arbitrary dimension d) for few important QCVV metrics. Second of all, we would like to point out that unexpectedly (at least for the authors) there are still interesting and non-trivial problems associated with the sole description of single qubit channels.

\section{Preliminaries}

From now on, since the gauge transformation acts in the same way on all gates from $\mathcal{G}$, we restrict our considerations to a single quantum channel $\mathcal{E}$ acting on $d \times d$ density matrices. The action of the channel, defined in terms of $L \leq d^{2}$ Kraus operators $K_{n}$,

$$
\mathcal{E}(\rho)=\sum_{n=1}^{L} K_{n} \rho K_{n}^{\dagger},
$$

leads to a representation of the superoperator by a square matrix of size $d^{2}$,

$$
\Phi=\sum_{n=1}^{L} K_{n} \otimes \bar{K}_{n},
$$

where $\bar{K}_{n}$ denotes the complex conjugate of $K_{n}$. Given an operator basis $\left\{B_{0}, \ldots, B_{d^{2}-1}\right\}$, such that $B_{0}=\mathbb{1} / \sqrt{d}$ and $\operatorname{Tr}\left(B_{i}^{\dagger} B_{j}\right)=\delta_{i j}$, the superoperator further acquires the block form

$$
\Phi=\left(\begin{array}{cc}
1 & 0 \\
k & T
\end{array}\right),
$$

provided that we assume the channel $\mathcal{E}$ to be trace preserving. Up to $d^{2}-1$ dimensional rotations, such basis is formed by the generalized Pauli matrices. The real matrix $T$ is supposed to act on generalized Bloch vectors of a quantum state, while the real vector $\boldsymbol{k}$ accounts for the translation of the center of of the set of quantum states and vanishes for unital maps. 
Real matrices with the block structure (5) and non-singular $T$ form a group $G$, being a subgroup of $G L_{d^{2}}(\mathbb{R})$, such that $A \in G$ if $A \in G L_{d^{2}}(\mathbb{R})$ and $A_{0 j}=\delta_{0 j}$ (trace preservation). If $T$ is singular, trace preservation defines a sub-semigroup of $d^{2}$-dimensional matrices. A natural requirement, saying that the reconstructed matrix $M$ from (1) respects trace preservation enforces gauge matrices to belong to $G$. One can verify this observation by testing the relevant condition

$$
\sum_{k, l} X_{0 l} A_{l k}\left(X^{-1}\right)_{k m}=\delta_{0 m}, \quad m=0, \ldots, d^{2}-1,
$$

for any $A$ in the block form (5). After multiplying the above vector by $X$ one finds

$$
X_{00} \delta_{0 m}+\sum_{l} X_{0 l} A_{l m}=X_{0 m}
$$

The unique solution of this equation (assuming that $X$ does not depend on $A$ ) is $X_{0 m}=X_{00} \delta_{0 m}$. We can set $X_{00}=1$ since for any number $\chi$ the matrices $X$ and $\chi X$ provide the same gauge transformation.

As a result of the above analysis, all gauge invariant information about the superoperator is stored in its eigenvalues, since

$$
\lambda_{m}(\Phi)=\lambda_{m}(M),
$$

for $m=0, \ldots, d^{2}-1$. By $\lambda_{m}(\cdot)$ we denote eigenvalues of a matrix and stick to the convention that $\lambda_{0}(\cdot)=1$, which is true for the block form (5). More formally, considering non-singular $T$, we say that only class functions (those which are constant on conjugacy classes for $G$, defined by the gauge) provide the way towards gauge invariant description of quantum gates.

Looking at the discussed problem from the perspective of theoretical quantum mechanics, we can see that the general aim is reduced to studies of spectrum of $\Phi$. Quite surprisingly, the question posed in that purely formal way, namely how much do we know about the spectrum of the superoperator corresponding to a CP TP map, has not been answered in a comprehensive manner. We know that [14]:

1. $\lambda_{0}(\Phi)=1$, due to trace preservation,

2. other $\lambda_{m}(\Phi)=1, m=1, \ldots, d^{2}-1$ are in general complex, though in practice they are either real or come in conjugate pairs (so that $\operatorname{det} \Phi$ and $\operatorname{Tr} \Phi$ are real),
3. All eigenvalues belong to the unit disk, $\left|\lambda_{m}(\Phi)\right| \leq 1$.

These general statements on the spectrum of stochastic quantum maps can be considered as an analogue of the Frobenius-Perron theorem [15], which concerns spectra of stochastic matrices.

Mathematical literature contains also more precise and refined results concerning the spectrum of quantum maps. A quantum operation $\Phi$ is called irreducible if there is no non-trivial face of the cone of quantum states invariant under $\Phi$. In such a case the leading eigenvalue $\lambda_{0}=1$ is non-degenerated $[16,17]$. Working with the Kraus representation (3) it is possible to show [18] that the map is irreducible if and only if the set of Kraus operators $\left\{K_{1}, \ldots K_{L}\right\}$ has no nontrivial common invariant subspace.

The structure of the peripheral spectrum, which contains all eigenvalues of $\Phi$ with modulus one, was studied by Groh in the eighties [19] and also more recently in context of quantum information [20, 21, 22]. A map is called primitive if the peripheral spectrum has no eigenvalues different than one. Operational criteria allowing one to establish whether a given map is primitive were established in [23]. For a primitive map with nondegenerated leading eigenvalue $\lambda_{0}=1$, the subleading eigenvalue $\lambda_{1}$ has modulus smaller than one, so the spectral gap, $\gamma:=1-\left|\lambda_{1}\right|$ is positive. The size of the spectral gap determines the quantum dynamics, as it yields an estimate for the rate with which any initial state converges to the single invariant state, $\rho_{0}$ associated with $\lambda_{0}$. In the case of a generic quantum map acting on a $d$-dimensional system the gap is typically large, as the modulus of the subleading eigenvalue $\left|\lambda_{1}\right|$ scales $[15,24]$ as $1 / d$.

\section{Gauge invariant information about complete positivity of a quantum chan- nel}

We now focus on our first question, namely how much information about complete positivity of a channel can be learned from the sole collection of eigenvalues of the superoperator. We shall consider the simplest, but fairly non-trivial case $d=$ 2 , and use the singular-value decomposition to represent $T=O_{1} \eta O_{2}^{T}$ with $\eta=\operatorname{diag}\left(\eta_{1}, \eta_{2}, \eta_{3}\right)$, being a diagonal matrix [25]. Since $O_{1}, O_{2} \in$ 
$S O(3)$, the real parameters $\eta_{l}$ can be negative. The singular values of $T$ are thus given by $s_{k}=$ $\left|\eta_{k}\right|$.

The only sensible way of comparison between arbitrary collections of singular values $\left(s_{i}\right)$ and eigenvalues $\left(\lambda_{i}\right)$ is by means of majorization relations (weak and log majorization respectively):

$$
\begin{gathered}
\sum_{i=1}^{k}\left|\lambda_{i}\right|^{\downarrow} \leq \sum_{i=1}^{k} s_{i}^{\downarrow}, \quad k=1, \ldots, N, \\
\prod_{i=1}^{k}\left|\lambda_{i}\right|^{\downarrow} \leq \prod_{i=1}^{k} s_{i}^{\downarrow}, \quad k=1, \ldots, N-1, \\
\prod_{i=1}^{N}\left|\lambda_{i}\right|^{\downarrow}=\prod_{i=1}^{N} s_{i}^{\downarrow},
\end{gathered}
$$

applicable to every $N \times N$ matrix. Traditionally, by " $\downarrow$ " we denote the decreasing order, so that $s_{k}^{\downarrow} \geq s_{l}^{\downarrow}$ for all $k \leq l$, and the same ordering is applied to the moduli of eigenvalues.

\subsection{Unital quantum channels}

We will first discuss the case of a unital channel, with vanishing translation vector $\boldsymbol{k}=0$, which is known to be completely positive if and only if the Fujiwara-Algoet conditions (FA) [26]

$$
1 \pm \eta_{3} \geq\left|\eta_{1} \pm \eta_{2}\right|
$$

are satisfied. There is no genuine relation between the numbers $\eta_{l}$ (here and later $l=1,2,3$ ) and the eigenvalues $\lambda_{l}(\Phi) \equiv \lambda_{l}(T)$, except from the determinant condition

$$
\operatorname{det} T=\eta_{1} \eta_{2} \eta_{3}=\lambda_{1} \lambda_{2} \lambda_{3}
$$

The determinant of $T$ will thus be of special interest for us later on. To simplify the notation, we omit inside the current section (whenever there is no confusion) the matrix argument of involved eigenvalues.

In order to move towards the goal assumed, we express the FA conditions in terms of the singular values equal to the absolute values of the parameters $\eta_{l}$. To this end, one needs to examine (see Table 1) all eight combinations of sign choices for the eta parameters. Put together, this analysis leads to the equivalent form of Eq. (10)

$$
\left\{\begin{array}{ll}
1 \pm s_{3} \geq\left|s_{1} \pm s_{2}\right| & \text { for } \operatorname{det} T \geq 0 \\
1 \pm s_{3} \geq\left|s_{1} \mp s_{2}\right| & \text { for } \operatorname{det} T \leq 0
\end{array}\right. \text {. }
$$

\begin{tabular}{|c|c|c|c|c|}
\hline$\eta_{1}$ & $\eta_{2}$ & $\eta_{3}$ & $\operatorname{sign}(\operatorname{det} T)$ & $1 \pm \eta_{3} \geq\left|\eta_{1} \pm \eta_{2}\right|$ \\
\hline \hline$+s_{1}$ & $+s_{2}$ & $+s_{3}$ & 1 & $1 \pm s_{3} \geq\left|s_{1} \pm s_{2}\right|$ \\
\hline$+s_{1}$ & $+s_{2}$ & $-s_{3}$ & -1 & $1 \mp s_{3} \geq\left|s_{1} \pm s_{2}\right|$ \\
\hline$+s_{1}$ & $-s_{2}$ & $+s_{3}$ & -1 & $1 \pm s_{3} \geq\left|s_{1} \mp s_{2}\right|$ \\
\hline$+s_{1}$ & $-s_{2}$ & $-s_{3}$ & 1 & $1 \mp s_{3} \geq\left|s_{1} \mp s_{2}\right|$ \\
\hline$-s_{1}$ & $+s_{2}$ & $+s_{3}$ & -1 & $1 \pm s_{3} \geq\left|s_{1} \mp s_{2}\right|$ \\
\hline$-s_{1}$ & $+s_{2}$ & $-s_{3}$ & 1 & $1 \mp s_{3} \geq\left|s_{1} \mp s_{2}\right|$ \\
\hline$-s_{1}$ & $-s_{2}$ & $+s_{3}$ & 1 & $1 \pm s_{3} \geq\left|s_{1} \pm s_{2}\right|$ \\
\hline$-s_{1}$ & $-s_{2}$ & $-s_{3}$ & -1 & $1 \mp s_{3} \geq\left|s_{1} \pm s_{2}\right|$ \\
\hline
\end{tabular}

Table 1: List of all sign combinations for the triple of eta parameters. Note that whenever the sign of $\operatorname{det} T$ is positive, the two " \pm " signs present in the FA condition (12) are the same, while they are reverted whenever $\operatorname{det} T$ is negative.

To obtain yet another, more friendly form of these conditions, we examine different orderings between the singular values. For example, if $s_{1} \geq s_{2} \geq s_{3}$ then the condition with positive determinant reduces to $1-\left(s_{1}+s_{2}+s_{3}\right)+2 s_{3} \geq 0$ and $1-\left(s_{1}+s_{2}+s_{3}\right)+2 s_{2} \geq 0$, for "+" and "-" case respectively. We can see that the first variant is more restrictive. In this way (going through all the orderings) one can replace (12) by

$$
1-s_{1}-s_{2}-s_{3}+2 \min \left\{s_{1} ; s_{2} ; s_{3}\right\} \geq 0,
$$

for $\operatorname{det} T \geq 0$ and

$$
1-s_{1}-s_{2}-s_{3} \geq 0,
$$

for $\operatorname{det} T \leq 0$. The two cases trivially coincide for $\operatorname{det} T=0$ since in this case $\min \left\{s_{1} ; s_{2} ; s_{3}\right\}=0$. We can also see that if $\operatorname{det} T>0$ it is possible to assign $s_{1}=s_{2}=s_{3}=1$ (for instance by $\eta_{1}=$ $\eta_{2}=\eta_{3}=1$ ), while if $\operatorname{det} T<0$ there is no such possibility. Due to arithmetic-geometric mean inequality one has $s_{1} s_{2} s_{3} \leq\left(s_{1}+s_{2}+s_{3}\right)^{3} / 27$. Therefore, assuming that the determinant is negative, from (13) we find that $s_{1} s_{2} s_{3} \leq 1 / 27$, and consequently $0 \geq \operatorname{det} T \geq-1 / 27$. This result was known before (see Corollary 10 from [20]) and it is interesting to add that (Example 4 from [20]) for every $d$, there exists a channel with $\operatorname{det} T=-(d+1)^{1-d^{2}}$ equal to $-1 / 27$ for $d=2$.

So far, we have managed to rewrite the FA conditions in terms of the singular values of the matrix $T$, supported by $\operatorname{det} T$ which being function of the eigenvalues is already in desired form. To go further, we need to relax (13) with the help of 
the majorization relations. From the weak majorization (9a) we find

$$
-s_{1}-s_{2}-s_{3} \leq-\left(\left|\lambda_{1}\right|+\left|\lambda_{2}\right|+\left|\lambda_{3}\right|\right),
$$

while due to log majorization $(9 \mathrm{~b}, 9 \mathrm{c})$ we get

$$
s_{N}^{\downarrow} \prod_{i=1}^{N-1} s_{i}^{\downarrow}=\left|\lambda_{N}\right|^{\downarrow} \prod_{i=1}^{N-1}\left|\lambda_{i}\right|^{\downarrow} \leq\left|\lambda_{N}\right|^{\downarrow} \prod_{i=1}^{N-1} s_{i}^{\downarrow},
$$

so that $s_{N}^{\downarrow} \leq\left|\lambda_{N}\right|^{\downarrow}$, and consequently

$$
\min \left\{s_{1} ; s_{2} ; s_{3}\right\} \leq \min \left\{\left|\lambda_{1}\right|,\left|\lambda_{2}\right|,\left|\lambda_{3}\right|\right\} .
$$

Making use of the above inequalities we finally obtain:

Theorem 1 If $\Phi$ is completely positive and unital, then

$$
1-\left(\left|\lambda_{1}\right|+\left|\lambda_{2}\right|+\left|\lambda_{3}\right|\right)+2 \min \left\{\left|\lambda_{1}\right|,\left|\lambda_{2}\right|,\left|\lambda_{3}\right|\right\} \geq 0,
$$

for $\lambda_{1} \lambda_{2} \lambda_{3} \geq 0$ and

$$
1-\left(\left|\lambda_{1}\right|+\left|\lambda_{2}\right|+\left|\lambda_{3}\right|\right) \geq 0,
$$

for $\lambda_{1} \lambda_{2} \lambda_{3}<0$.

The theorem, as giving necessary criteria of complete positivity, asserts that the range of eigenvalues of $T$ is contained in the set characterized by the above inequalities. However if $T$ is a normal matrix, so that its singular values are the same as moduli of the eigenvalues, the criteria also become sufficient.

In Section 5 we show that if a collection of numbers $\left\{\lambda_{1}, \lambda_{2}, \lambda_{3}\right\}$ satisfies the conditions (17), and moreover all $\lambda_{i}$ are real, or $\lambda_{1} \in \mathbb{R}$ and $\lambda_{2}=\bar{\lambda}_{3}$, then there exists a unital quantum channel with eigenvalues $\left\{1, \lambda_{1}, \lambda_{2}, \lambda_{3}\right\}$.

\subsection{Non-unital channels}

After we derived the gauge invariant necessary criteria for complete positivity of unital quantum channels, an obvious way of continuation shall concern the general case of an arbitrary translation vector $\boldsymbol{k} \neq 0$. For $d=2$, the relevant generalization of the FA conditions was known long ago [27]. The requirement of gauge invariance, however, cannot be straightforwardly imposed on this result, because the gauge brings problems of conceptual nature. It is clear, that the eigenvalues of $\Phi$ are independent of $\boldsymbol{k}$. In other words, the whole, a priori information about the vector $\boldsymbol{k}$ is lost due to the gauge symmetry. Therefore, one is not able to derive conditions for complete positivity different than (17). Instead, one can hope that:

1. the necessary criteria Eq. (17) remain valid for non-unital channels,

2. one can a posteriori gain some knowledge about $\boldsymbol{k}$, by imposing complete positivity.

It turns out, that both expectations are met.

The first one can be best seen from the alternative shape of criteria from [27], derived recently in [28]. In their Theorem 4.1 it is explicitly shown that for any translation $\boldsymbol{k}$, the inequalities (10) must be satisfied, and there is an additional (single) inequality involving $\boldsymbol{k}$. In other words, since the conditions (10) hold in general, the same applies to (17).

The extra condition [given in [28] also as Eqs. (37) and (38)] involving $\boldsymbol{k}$ can then serve to restrict the range of this vector, which in the gauge invariant picture is unknown. According to Eq. (38) from [28]

$$
\|\boldsymbol{k}\|^{2} \leq 1-\sum_{i=1}^{3} \eta_{i}^{2}+2 \eta_{1} \eta_{2} \eta_{3} \equiv 1-\sum_{i=1}^{k} s_{i}^{2}+2 \operatorname{det} T,
$$

with $\|\cdot\|$ being the standard Euclidean norm. Due to the majorization property (9a) we immediately obtain the gauge invariant bound:

Corollary 1 Whenever $\Phi$ is completely positive, then

$$
\|\boldsymbol{k}\|^{2} \leq 1-\left|\lambda_{1}\right|^{2}-\left|\lambda_{2}\right|^{2}-\left|\lambda_{3}\right|^{2}+2 \lambda_{1} \lambda_{2} \lambda_{3} .
$$

The above bound, which restricts the norm of $\boldsymbol{k}$ for completely positive channels seems relatively strong. For instance, the special case $\lambda_{1}=\lambda_{2}=$ $\lambda_{3}=1$, implies $\boldsymbol{k}=0$, so the channel $\Phi$ is forced to be unital.

The remaining condition Eq. (37) from [28], in its explicit form $Z(\eta)=$

$$
\|\boldsymbol{k}\|^{4}-2\|\boldsymbol{k}\|^{2}-2 \sum_{i=1}^{3} \eta_{i}^{2}\left(2 k_{i}^{2}-\|\boldsymbol{k}\|^{2}\right)+q(\eta) \geq 0,
$$

with

$$
\begin{aligned}
q(\eta)= & \left(1+\eta_{1}+\eta_{2}+\eta_{3}\right)\left(1+\eta_{1}-\eta_{2}-\eta_{3}\right) \times \\
& \left(1-\eta_{1}+\eta_{2}-\eta_{3}\right)\left(1-\eta_{1}-\eta_{2}+\eta_{3}\right),
\end{aligned}
$$


does not allow for a straightforward characterization in terms of the eigenvalues of $\Phi$. One can however use the same strategy as before to pass from the eta parameters to the singular values. The condition (20) in terms of $s_{k}$ reads:

$$
\left\{\begin{array}{ll}
Z(s) \geq 0 & \text { for } \operatorname{det} T \geq 0 \\
Z(s)-16 \operatorname{det} T \geq 0 & \text { for } \operatorname{det} T \leq 0
\end{array},\right.
$$

with $Z(\cdot)$ defined in Eq. 20. Given the three eigenvalues of $\Phi$ one can (numerically) optimize the condition with respect to the singular values, taking the majorization relations (9) as meaningful constraints.

\section{Gauge invariant bounds on QCVV metrics}

In the previous section we were concerned with a formal characterization of complete positivity (for $d=2$ ) by means of eigenvalues of the superoperator. Due to the relation (8), the obtained results allow for a direct use of the matrix $M$ which is the GST-reconstructed (from measurement data) superoperator subject to the gauge (1).

One of the main practical goals of GST is verification, whether an experimentally implemented quantum channel is a faithful representation of a presumed specification (a target gate). To this end one can evaluate various, relevant metrics such as the diamond distance or average gate fidelity. A natural question to be asked is how much about such metrics can one say using the eigenvalues of the superoperator (so that one can only use the information from $M$ )? Our aim now is to sketch an answer to that question.

In what follows, we will be concerned with a case of an arbitrary dimension $d$ and the following three metrics:

- Average gate fidelity [8],

$$
\mathcal{F}_{\text {avg }}(\mathcal{E})=\int d \psi\langle\psi|\mathcal{E}(|\psi\rangle\langle\psi|)| \psi\rangle,
$$

also related to the average error rate $r(\mathcal{E})=$ $1-\mathcal{F}_{\text {avg }}(\mathcal{E})$,

- Unitarity [37] $u(\mathcal{E})=$

$$
\frac{d}{d-1} \int d \psi \operatorname{Tr}\left[\mathcal{E}^{\prime}(|\psi\rangle\langle\psi|)^{\dagger} \mathcal{E}^{\prime}(|\psi\rangle\langle\psi|)\right],
$$

with $\mathcal{E}^{\prime}(\rho)=\mathcal{E}(\rho)-[\operatorname{Tr} \mathcal{E}(\rho)] \mathbb{1} / \sqrt{d}$,
- Diamond norm [9], equivalent to completely bounded trace norm,

$$
\epsilon_{\diamond}(\mathcal{E})=\frac{1}{2} \sup _{\sigma}\left\|\left(\mathbb{1}_{d} \otimes \mathcal{E}\right) \sigma\right\|_{1},
$$

with $\|\cdot\|_{1}$ being the trace norm. In general the supremum is performed over the entire set of the extended states of an arbitrary dimension, but in analogy to the property of complete positivity it is sufficient to take the size of the ancillary system to be equal to the size $d$ of the principal system [9], so in our case the extended state $\sigma$ has dimension $d^{2}$.

The average gate fidelity is a standard measure suitable for error quantification in quantum gates, since it can be efficiently estimated experimentally with the help of randomized benchmarking. This big practical advantage is shared with unitarity, the measure designed for coherent noise. The diamond distance between two channels $\epsilon_{\diamond}\left(\mathcal{E}_{1}-\mathcal{E}_{2}\right)$ is of importance for thresholds of fault tolerance in quantum computing. However, no efficient protocol to measure this quantity is known, computation of this distance is rather involved and requires semi-definite programming [29]. Analytical results were obtained in the asymptotic case only [30], for which the diamond distance between two random channels was derived.

We shall start with the following, positively surprising observation.

Proposition 1 The average gate fidelity $\mathcal{F}_{\text {avg }}(\mathcal{E})$ is gauge invariant.

Proof. We resort to the well known formula for average gate fidelity [31, 32, 33]

$$
\mathcal{F}_{\text {avg }}(\mathcal{E})=\frac{\operatorname{Tr} \Phi+d}{d(d+1)} .
$$

According to (8), $\operatorname{Tr} \Phi=\operatorname{Tr} M$. Note that, even though expressions for higher order moments (like the variance) of gate fidelity $[34,35]$ or the minimal fidelity [36] have been studied, it is not possible to provide their gauge invariant forms. This observation gives the average gate fidelity a sort of a unique meaning.

Having the gauge invariance of the first metric established, we can now move to the second and the third one, which definitely are not invariant. Note however, that these quantities can 
be bounded in terms of the average error rate,

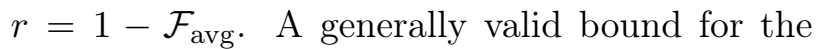
unitarity,

$$
u(\mathcal{E}) \geq\left[1-\frac{d}{d-1} r(\mathcal{E})\right]^{2},
$$

was obtained in Proposition 8 in [37], while the following bounds for the diamond norm

$$
\frac{d+1}{d} r(\mathcal{E}) \leq \epsilon_{\diamond}\left(\mathcal{E}-\mathbb{1}_{d}\right) \leq \sqrt{d(d+1) r(\mathcal{E})},
$$

were established as Proposition 9 in [13]. The bound (27) is saturated if and only if the matrix $T$ in (5) is a diagonal scalar matrix.

Since $r(\mathcal{E})$ is gauge invariant the above inequalities provide ready-to-go estimates for both the diamond distance and the unitarity. More involved lower and upper bounds for the diamond distance given as functions of average error rate and unitarity have been recently derived in $[38,39]$. Note that these upper bounds, after one sets $u(\mathcal{E})=1$, are again function of the gauge invariant average error rate. Here, we shall only quote the sharpest among the lower bounds [39]

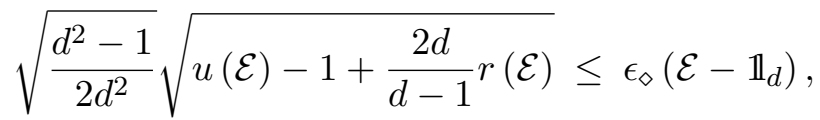

Quite obviously the unitarity, which is not gauge invariant, can be lower-bounded by (27). To make a range of available gauge-invariant options a bit wider, we shall prove the following

Proposition 2 Unitarity is lower-bounded

$$
u(\mathcal{E}) \geq \frac{\sum_{k}\left|\lambda_{k}(\Phi)\right|^{2}-d}{d(d-1)},
$$

with $\lambda_{k}(\Phi)$, for $k=0, \ldots, d^{2}-1$, being the eigenvalues of $\Phi$. The bound is tight whenever the channel is unitary.

Proof. By virtue of Proposition 9 from [37] one has

$$
u(\mathcal{E})=\frac{\operatorname{Tr}\left[\Phi^{\dagger} \Phi\right]-1-\|\boldsymbol{k}\|^{2}}{d^{2}-1},
$$

with $\boldsymbol{k}$ being the non-unitality vector defined in (5). Moreover, Proposition 6 from [37] establishes the bound $\|\boldsymbol{k}\|^{2} \leq(d-1)[1-u(\mathcal{E})]$, which can be directly applied to the previous equation, leading to

$$
u(\mathcal{E}) \geq \frac{\operatorname{Tr}\left[\Phi^{\dagger} \Phi\right]-1-(d-1)[1-u(\mathcal{E})]}{d^{2}-1},
$$

We can solve the last inequality for $u(\mathcal{E})$ getting

$$
u(\mathcal{E}) \geq \frac{\operatorname{Tr}\left[\Phi^{\dagger} \Phi\right]-d}{d(d-1)}
$$

The term $\operatorname{Tr}\left[\Phi^{\dagger} \Phi\right]$ is the sum of squared singular values of $\Phi$. Since the singular values always majorize (see Eq. 9a) the moduli of eigenvalues of a given matrix (and second power is a Shur-convex function) we obtain the desired bound (30). Both (27) and (30) can further be used to provide gauge invariant lower bounds for (29).

\section{Discussion}

The motivation of this paper stems from the similarity (gauge) relation (1) being of relevance for gate set tomography. If one takes the limitations brought by the gauge seriously, one is directed towards gauge invariant ways of inferring conclusions. However, contrarily to the standard case of field theories in physics, while passing to gauge invariant description we lose accuracy. Even though, one is allowed to argue that the criteria and bounds derived in Sec. 3 and Sec. 4 respectively could potentially be improved, we shall stress that some loss of information is unavoidable. To make it evident, we show the following result valid for qubit gates.

As explained in the preliminary part, the eigenvalues of $\Phi$ are either real or come in complexconjugate pairs. For $d=2$ this implies that either all three non-trivial eigenvalues are real or (without loss of generality): $\lambda_{1}(\Phi)=x \in \mathbb{R}$ and $\lambda_{2}(\Phi)=z, \lambda_{3}(\Phi)=\bar{z}$ with $z \in \mathbb{C}$ and $\operatorname{Im} z \neq 0$. In the latter case, the CP conditions (17), which as explained in Sec. 3.2 are valid for all qubit channels (not only for unital ones), simplify to the form

$$
\left\{\begin{array}{ll}
1-(x+2|z|)+2 \min \{x,|z|\} \geq 0 & \text { for } x \geq 0 \\
1-(|x|+2|z|) \geq 0 & \text { for } x \leq 0
\end{array} .\right.
$$

It can easily be deduced, that since $|x| \leq 1$ and $|z| \leq 1$ the above, in fact, reduces to a single, basic inequality

$$
|z| \leq \frac{1+x}{2}
$$

valid for any one-qubit unital quantum operation with two complex eigenvalues of the corresponding superoperator. Possible values of the eigenvalue $z$, for a given $x$, are plotted in Fig. (1). 


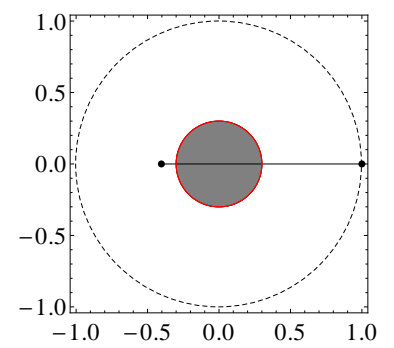

(a) $x=-0.4$

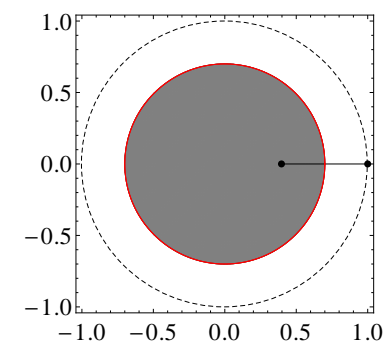

(b) $x=0.4$
Figure 1: The range of complex eigenvalues for qubit quantum channels. In the grey region we plot possible values of $z$ in the case when eigenvalues of a qubit channel are in the form $\{1, x, z, \bar{z}\}$, where $x$ is marked at the real axis.

In the case of all three eigenvalues are real the complete positivity conditions (17) are equivalent to the following

$$
\begin{aligned}
& 1+\lambda_{1}+\lambda_{2}+\lambda_{3} \geq 0, \\
& 1+\lambda_{1}-\lambda_{2}-\lambda_{3} \geq 0, \\
& 1-\lambda_{1}+\lambda_{2}-\lambda_{3} \geq 0, \\
& 1-\lambda_{1}-\lambda_{2}+\lambda_{3} \geq 0 .
\end{aligned}
$$

The above states, that if a qubit channel, regardless of whether it is unital or not, has all real eigenvalues, they must lay in a tetrahedron defined in (36).

Now, let us define two channels, namely: a classical doubly stochastic channel, described by a superoperator in form of an extended bistochastic matrix,

$$
S_{a}=\left(\begin{array}{cccc}
a & 0 & 0 & 1-a \\
0 & 0 & 0 & 0 \\
0 & 0 & 0 & 0 \\
1-a & 0 & 0 & a
\end{array}\right) \quad \text { for } 0 \leq a \leq 1,
$$

and a unitary channel with its eigenvectors forming the computational basis

$$
\Psi_{\alpha}=\left(\begin{array}{cc}
1 & 0 \\
0 & e^{i \alpha}
\end{array}\right) \otimes\left(\begin{array}{cc}
1 & 0 \\
0 & e^{-i \alpha}
\end{array}\right)=\left(\begin{array}{cccc}
1 & 0 & 0 & 0 \\
0 & e^{-i \alpha} & 0 & 0 \\
0 & 0 & e^{i \alpha} & 0 \\
0 & 0 & 0 & 1
\end{array}\right) .
$$

We are in position to prove the following

Proposition 3 Let $\Phi$ be a superoperator of a qubit unital channel characterized by eigenvalues $\left\{1, \lambda_{1}, \lambda_{2}, \lambda_{3}\right\}$ if:

- eigenvalues are in the form $\{1, x, z, \bar{z}\}$ with $\operatorname{Im} z \neq 0$ then $\Phi$ is gauge-equivalent to a mixture of a classical map and a unitary transformation,

$$
\Phi_{p, a, \alpha}=p S_{a}+(1-p) \Psi_{\alpha},
$$

- all eigenvalues are real, then $\Phi$ is gaugeequivalent to a normal superoperator

$$
\Xi_{\lambda}=\frac{1}{2}\left(\begin{array}{cccc}
1+\lambda_{1} & 0 & 0 & 1-\lambda_{1} \\
0 & \lambda_{2}+\lambda_{3} & \lambda_{3}-\lambda_{2} & 0 \\
0 & \lambda_{3}-\lambda_{2} & \lambda_{2}+\lambda_{3} & 0 \\
1-\lambda_{1} & 0 & 0 & 1+\lambda_{1}
\end{array}\right) .
$$

Proof. The superoperator (39) has the eigenvalues: $\left\{1,1-2 p(1-a),(1-p) e^{-i \alpha},(1-p) e^{i \alpha}\right\}$. They will be equal to those of $\Phi$, provided that: $p=1-|z|, a=\frac{x-2|z|+1}{2-2|z|}$ and $\alpha=\arg (z)$. This is enough to assure that $\Phi$ and $\Phi_{p, a, \alpha}$ are connected by a similarity relation (1). One shall only check that the mixture is proper, namely that $0 \leq p, a \leq 1$. The first requirement $0 \leq p \leq 1$ is trivial since $|z| \leq 1$. The second one is also simple, i.e.

$$
\begin{aligned}
& \frac{x-2|z|+1}{2-2|z|} \geq 0 \Longleftrightarrow|z| \leq \frac{1+x}{2}, \\
& \frac{x-2|z|+1}{2-2|z|} \leq 1 \Longleftrightarrow x \leq 1 .
\end{aligned}
$$

These requirements are satisfied by virtue of our assumptions, in particular, due to the complete positivity condition (35). In the case of real eigenvalues the proof follows by a direct inspection.

The above statement is an emanation of the information loss associated with the gaugeinvariant description. Having in our disposal the eigenvalues of $\Phi$ and nothing more, we are in principle unable to differentiate between the actual quantum channel and e.g. the mixture (39), even if the original channel is clearly distinct from it.

To shortly conclude, our aim was to check, how much of relevant information about a quantum channel can be obtained from a collection of the eigenvalues of the associated superoperator. As one might not necessarily expect particularly surprising answers to the above question, we shall emphasize practical relevance (within GST) of the problem studied. It is clear, that even though the eigenvalues alone contain a substantial amount of valuable content, the limitation imposed by the GST gauge invariance treated seriously is quite severe. It thus seems the GST protocol would gain a lot, provided that one is able to experimentally infer anything about the gauge degrees of freedom. Quite obviously, since the GST gauge is not put by the nature on the same footing as gauge symmetries of fundamental interaction, deeper studies aiming at providing a routine to fix the gauge in a possibly cleanest way 
(maybe not necessarily as the most optimistic one from the QCVV perspective) are worth further research.

On the positive side we shall emphasize that the average gate fidelity or the average error rate are completely specified by the eigenvalues of the superoperator, accessible by the gate set tomography procedure. This fact, shall potentially let these quantifiers be even more useful than they have been by now.

\section{Acknowledgments}

We would like to thank David Gross for fruitful discussions and his support in proving Proposition 2. Ł.R. acknowledges financial support by grant number $2014 / 13 / \mathrm{D} / \mathrm{ST} 2 / 01886$ of the National Science Center, Poland. Research in Cologne has been supported by the Excellence Initiative of the German Federal and State Governments (Grants ZUK 43 \& 81), the ARO under contract W911NF-14-1- 0098 (Quantum Characterization, Verification, and Validation), and the DFG projects GRO 4334/1,2 (SPP1798 CoSIP). Z.P. acknowledges the support by the Polish National Science Center under the Project Number 2016/22/E/ST6/00062. K.Ż. acknowledges the support by the Polish National Science Center under the Project Number DEC2015/18/A/ST2/00274 and by the John Templeton Foundation under the Project No. 56033.

\section{References}

[1] R. Blume-Kohout, et al., Robust, self-consistent, closed-form tomography of quantum logic gates on a trapped ion qubit, arXiv:1310.4492 (2013)

[2] D. Kim, et al., Microwave-driven coherent operation of a semiconductor quantum dot charge qubit, Nat. Nanotechnology 10, 243 (2015).

[3] D. Greenbaum, Introduction to Quantum Gate Set Tomography, arXiv:1509.02921 (2015).

[4] J. F. Poyatos, J. I. Cirac, and P. Zoller, Complete Characterization of a Quantum Process: The Two-Bit
Quantum Gate, Phys. Rev. Lett. 78, 390 (1997).

[5] S. T. Merkel, et al., Self-consistent quantum process tomography, Phys. Rev. A 87, 062119 (2013).

[6] T. Baumgratz, D. Gross, M. Cramer, and M. B. Plenio, Scalable Reconstruction of Density Matrices, Phys. Rev. Lett. 111, 020401 (2013).

[7] R. Blume-Kohout, et al., Demonstration of qubit operations below a rigorous fault tolerance threshold with gate set tomography, Nat. Commun. 8, 14485 (2017).

[8] M. A. Nielsen and I. L. Chuang, Quantum Computation and Quantum Information, Cambridge University Press, Cambridge, 2000

[9] A. Y. Kitaev, A. Shen, and M. N. Vyalyi, Classical and quantum computation, Vol. 47 (American Mathematical Society, 2002).

[10] J. Emerson, R. Alicki, K. Życzkowski, Scalable Noise Estimation with Random Unitary Operators, J. Opt. B: Quantum Semiclass. Opt. 7, S347 (2005).

[11] J. Emerson, et al., Symmetrized Characterization of Noisy Quantum Processes, Science 317, 1893 (2007).

[12] E. Knill, et al., Randomized benchmarking of quantum gates, Phys. Rev. A 77, 012307 (2008).

[13] J. J. Wallman and S. T. Flammia, Randomized benchmarking with confidence, New J. Phys. 16, 103032 (2014).

[14] K. Życzkowski and I. Bengtsson, On duality between quantum maps and quantum states, Open Sys. \& Information Dyn. 11, 3 (2004).

[15] W. Bruzda, V. Cappellini, H.-J. Sommers, and K. Życzkowski, Random Quantum Operations, Phys. Lett. A 373, 320 (2009). 
[16] D. Evans, R. Hoegh-Krohn, Spectral properties of positive maps on $C_{*}-$ algebras, J. London Math. Soc. 17, 345 (1978).

[17] A. Jamiołkowski, On applications of PI-algebras in the analysis of quantum channels, Int. J. Quantum Inf. 10, 1241007 (2012).

[18] D. R. Farenick, Irreducible positive linear maps on operator algebras, Proc. AMS 124, 3381, (1996).

[19] U. Groh, The peripheral point spectrum of Schwarz operators on $C_{*}$ algebras, Math. Z. 176, 311 (1981).

[20] M. M. Wolf and J. I. Cirac, Dividing Quantum Channels, Commun. Math. Phys. 279, 147 (2008).

[21] M. M. Wolf and D. Perez-Garcia, The inverse eigenvalue problems for quantum channels, preprint arXiv:1005.4545, (2010).

[22] M. Rahaman, Multiplicative Properties of Quantum Channels, J. Phys. A: Math. Theor. 50, 345302 (2017).

[23] M. Sanz, D. Perez-Garcia, M. M. Wolf, and J. I. Cirac, A quantum version of Wielandt's inequality, IEEE Trans. Inf. Theory 56, 4668 (2010).

[24] W. Bruzda, M. Smaczyński, V. Cappellini, H.-J. Sommers, K. Życzkowski, Universality of spectra for interacting quantum chaotic systems, Phys. Rev. E 81, 066209 (2010).

[25] I. Bengtsson and K. Życzkowski, Geometry of Quantum States, II edition, Cambridge University Press, Cambridge, 2017.

[26] A. Fujiwara and P. Algoet, Oneto-one parametrization of quantum channels, Phys. Rev. A 59, 3290 (1999).

[27] M. B. Ruskai, S. Szarek, and E. Werner, An analysis of completely positive trace-preserving maps on
$\mathscr{M}_{2}$, Linear Algebra Appl. 347, 159 (2002).

[28] D. Braun, O. Giraud, I. Nechita, C. Pellegrini, and M. Znidaric, A universal set of qubit quantum channels, J. Phys. A 47, 135302 (2014).

[29] J. Watrous, Simpler semidefinite programs for completely bounded norms, Chicago J. of Th. Comp. Sci. 2013, 8 (2013).

[30] I. Nechita, Z. Puchała, Ł. Pawela, and K. Życzkowski Almost all quantum channels are equidistant, arXiv:1612.00401 (2016).

[31] M. A. Nielsen, A simple formula for the average gate fidelity of a quantum dynamical operation, Phys. Lett. A 303, 249 (2002).

[32] M. D. Bowdrey, D. K. L. Oi, A. J. Short, K. Banaszek, and J. A. Jones, Fidelity of single qubit maps, Phys. Lett. A. 294, 258 (2002).

[33] M. Horodecki, P. Horodecki, and R. Horodecki, General teleportation channel, singlet fraction, and quasidistillation, Phys. Rev. A 60, 1888 (1999).

[34] E. Magesan, R. Blume-Kohout, and J. Emerson, Gate fidelity fluctuations and quantum process invariants, Phys. Rev. A 84, 012309 (2011).

[35] L. H. Pedersen, N. M. Møller, and K. Mølmer, The distribution of quantum fidelities, Phys. Lett. A 372, 7028 (2011).

[36] N. Johnston and D. W. Kribs, Quantum gate fidelity in terms of Choi matrices, J. Phys. A 44, 495303 (2011).

[37] J. Wallman, C. Granade, R. Harper, and S. T. Flammia, Estimating the Coherence of Noise, New J. Phys. 17, 113020 (2015).

[38] R. Kueng, D. M. Long, A. C. Doherty, and S. T. Flammia, Comparing Experiments to the Fault- 
Tolerance Threshold, Phys. Rev.

Lett. 117, 170502 (2016).

[39] J. Wallman, Bounding experimental quantum error rates relative to fault-tolerant thresholds, arXiv:1511.00727v2 (2016). 